\title{
MStream: Fast Anomaly Detection in Multi-Aspect Streams
}

\author{
Siddharth Bhatia \\ National University of Singapore \\ Singapore \\ siddharth@comp.nus.edu.sg
}

\author{
Arjit Jain \\ IIT Bombay \\ India \\ arjit@cse.iitb.ac.in
}

\author{
Pan Li \\ Purdue University \\ United States \\ panli@purdue.edu
}

\author{
Ritesh Kumar \\ IIT Kanpur \\ India \\ riteshk@iitk.ac.in
}

\author{
Bryan Hooi \\ National University of Singapore \\ Singapore \\ bhooi@comp.nus.edu.sg
}

\begin{abstract}
Given a stream of entries in a multi-aspect data setting i.e., entries having multiple dimensions, how can we detect anomalous activities in an unsupervised manner? For example, in the intrusion detection setting, existing work seeks to detect anomalous events or edges in dynamic graph streams, but this does not allow us to take into account additional attributes of each entry. Our work aims to define a streaming multi-aspect data anomaly detection framework, termed MSTREAM which can detect unusual group anomalies as they occur, in a dynamic manner. MSTREAM has the following properties: (a) it detects anomalies in multi-aspect data including both categorical and numeric attributes; (b) it is online, thus processing each record in constant time and constant memory; (c) it can capture the correlation between multiple aspects of the data. MSTREAM is evaluated over the KDDCUP99, CICIDS-DoS, UNSW-NB 15 and CICIDS-DDoS datasets, and outperforms state-of-the-art baselines.
\end{abstract}

\section{CCS CONCEPTS}

- Computing methodologies $\rightarrow$ Anomaly detection; Online learning settings; • Security and privacy $\rightarrow$ Intrusion detection systems.

\section{KEYWORDS}

Anomaly Detection, Multi-Aspect Data, Stream, Intrusion Detection

ACM Reference Format:

Siddharth Bhatia, Arjit Jain, Pan Li, Ritesh Kumar, and Bryan Hooi. 2021. MSTream: Fast Anomaly Detection in Multi-Aspect Streams. In Proceedings of the Web Conference 2021 (WWW'21), April 19-23, 2021, Ljubljana, Slovenia. ACM, New York, NY, USA, 12 pages. https://doi.org/10.1145/3442381.3450023

\section{INTRODUCTION}

Given a stream of entries (i.e. records) in multi-aspect data (i.e. data having multiple features or dimensions), how can we detect anomalous behavior, including group anomalies involving the sudden appearance of large groups of suspicious activity, in an unsupervised manner?

This paper is published under the Creative Commons Attribution 4.0 International (CC-BY 4.0) license. Authors reserve their rights to disseminate the work on their personal and corporate Web sites with the appropriate attribution.

WWW'21, April 19-23, 2021, Ljubljana, Slovenia

() 2021 IW3C2 (International World Wide Web Conference Committee), published under Creative Commons CC-BY 4.0 License.

ACM ISBN 978-1-4503-8312-7/21/04

https://doi.org/10.1145/3442381.3450023
In particular, we focus on an important application of anomaly detection to intrusion detection in networks. In this application, we want to design algorithms that monitor a stream of records, each of which represents a single connection (or 'flow') over the network. We aim to detect multiple types of suspicious activities, such as denial of service or port scanning attacks, whereby attackers make a large number of connections to a target server to make it inaccessible or to look for vulnerabilities.

Recent intrusion detection datasets typically report tens of features for each individual flow, such as its source and destination IP, port, protocol, average packet size, etc. This makes it important to design approaches that can handle multi-aspect data. In addition, to effectively guard against attacks, it is important for our algorithm to process the data in a streaming manner, so that we can quickly report any attack in real-time, as soon as it arrives.

Some existing approaches for this problem aim to detect point anomalies, or individually unusual connections. However, as this ignores the relationships between records, it does not effectively detect large and suddenly appearing groups of connections, as is the case in denial of service and other attacks. For detecting such groups, there are also existing methods based on dense subgraph detection [4] as well as dense subtensor detection [63, 65]. However, these approaches are generally designed for datasets with a smaller number of dimensions, thus facing significant difficulties scaling to our dataset sizes. Moreover, they treat all variables of the dataset as categorical variables, whereas our approach can handle arbitrary mixtures of categorical variables (e.g. source IP address) and numerical variables (e.g. average packet size).

Hence, in this work, we propose MSTREAM, a method for processing a stream of multi-aspect data that detects group anomalies, i.e. the sudden appearance of large amounts of suspiciously similar activity. Our approach naturally allows for similarity both in terms of categorical variables (e.g. a small group of repeated IP addresses creating a large number of connections), as well as in numerical variables (e.g. numerically similar values for average packet size).

MSTREAM is a streaming approach that performs each update in constant memory and time. This is constant both with respect to the stream length as well as in the number of attribute values for each attribute: this contrasts with tensor decomposition-based approaches such as STA and dense subtensor-based approaches such as DenseAlert, where memory usage grows in the number of possible attribute values. To do this, our approach makes use of locality-sensitive hash functions (LSH), which process the data 
in a streaming manner while allowing connections which form group anomalies to be jointly detected, as they consist of similar attribute values and hence are mapped into similar buckets by the hash functions. Finally, we demonstrate that the anomalies detected by MSTREAM are explainable.

To incorporate correlation between features, we further propose MStreAm-PCA, MStreAm-IB and MStreAm-AE which leverage Principal Component Analysis (PCA), Information Bottleneck (IB), and Autoencoders (AE) respectively, to map the original features into a lower-dimensional space and then execute MSTREAM in this lower-dimensional space. MSTREAM-AE is shown to provide better anomaly detection performance while also improving speed compared to MSTREAM, due to its lower number of dimensions.

In summary, the main contributions of our approach are:

(1) Multi-Aspect Group Anomaly Detection: We propose a novel approach for detecting group anomalies in multiaspect data, including both categorical and numeric attributes.

(2) Streaming Approach: Our approach processes the data in a fast and streaming fashion, performing each update in constant time and memory.

(3) Effectiveness: Our experimental results show that MSTREAM outperforms baseline approaches.

Reproducibility: Our code and datasets are publicly available at https://github.com/Stream-AD/MStream.

\section{RELATED WORK}

Our work is closely related to areas like anomaly detection on graphs $[1,5-7,20,33,34,38,49-51,58,68,75,78]$, graph and stream classification [10, 28, 42-44, 72], and outlier detection on streams $[35,40,57,64,73,74]$. In this section, we limit our review only to previous approaches for detecting anomalies on edge-streams, tensors and multi-aspect data. See [16] for an extensive survey on tensor-based anomaly detection.

Anomaly Detection in Edge Streams uses as input a stream of edges over time. We categorize them according to the type of anomaly detected.

- Anomalous node detection: Given an edge stream, [76] detects nodes whose egonets suddenly and significantly change.

- Anomalous subgraph detection: Given an edge stream, DenseAlert [63] identifies dense subtensors created within a short time.

- Anomalous edge detection: RHSS [54] focuses on sparselyconnected parts of a graph, while SEDANSPOT [14] identifies edge anomalies based on edge occurrence, preferential attachment, and mutual neighbors. MIDAs [4] identifies microcluster based anomalies, or suddenly arriving groups of suspiciously similar edges.

Anomaly Detection in Multi-Aspect Data Streams uses as input a stream of multi-aspect data records over time. Each multiaspect data record can also be considered as an edge of an attributed graph having multiple attributes. Therefore, in addition to detecting anomalies in multi-aspect data streams, the following approaches can also detect anomalies in edge streams.
- Score Plot based: Score Plots are obtained from tensor decomposition which are then analyzed manually or automatically for anomaly detection. These score plots can be onedimensional: [45], multi-dimensional: MALSpOT [36] or timeseries [46].

- Histogram based: MASTA [15] uses histogram approximation to analyze tensors. It vectorizes the whole tensor and simultaneously segments into slices in each mode. The distribution of each slice is compared against the vectorized tensor to identify anomalous slices.

- Tensor decomposition based: Tensor decomposition methods such as [27] can be used to find anomalies. [79] and STA [65] are streaming algorithms for CPD and Tucker decompositions. STenSr [60] models the tensor stream as a single incremental tensor for representing the entire network, instead of dealing with each tensor in the stream separately. [30] uses subspace learning in tensors to find anomalies. STA monitors the streaming decomposition reconstruction error for each tensor at each time instant and anomalies occur when this error goes beyond a pre-defined threshold. However [63] shows limited accuracy for dense-subtensor detection based on tensor decomposition.

- Dense subtensor detection based: Dense-subtensor detection has been used to detect anomalies in M-Zoom [61], D-CuBE [62], [37] and CrossSpot [23] but these approaches consider the data as a static tensor. DenseAlert [63] is a streaming algorithm to identify dense subtensors created within a short time.

Other Approaches for Anomaly Detection can typically be used in multi-aspect settings by converting categorical attributes to numerical ones e.g. using one-hot encoding. Elliptic Envelope [56] fits an ellipse to the normal data points by fitting a robust covariance estimate to the data. Local Outlier Factor (LOF) [8] estimates the local density at each point, then identifies anomalies as points with much lower local density than their neighbors. Isolation Forest (I-Forest) [32] constructs trees by randomly selecting features and splitting them at random split points, and then defines anomalies as points which are separated from the rest of the data at low depth values. Random Cut Forest (RCF) [19] improves upon isolation forest by creating multiple random cuts (trees) of data and constructs a forest of such trees to determine whether a point is anomalous or not.

Recently, deep learning approaches for anomaly detection in multi-aspect data have also been proposed. DAGMM [81] learns a Gaussian Mixture density model (GMM) over a low-dimensional latent space produced by a deep autoencoder. [24] use metric learning for anomaly detection. Deep Structured Energy-based Model for Anomaly Detection (DSEBM) [77] trains deep energy models such as Convolutional and Recurrent EBMs using denoising score matching instead of maximum likelihood, for performing anomaly detection. More recently, methods like APAE [17], MEG [29] and Fence GAN [41] have been successfully used to detect anomalies.

For the task of Intrusion Detection [2, 3, 18, 70, 71, 80], a variety of different approaches have been used in the literature including Ensemble methods [53], Feature Selection [52], Fuzzy Neural Networks [13], Kernel Methods [67], Random Forests [21], and deep 
Table 1: Comparison of relevant multi-aspect anomaly detection approaches.

\begin{tabular}{|c|c|c|c|c|c|c|c|c|c|}
\hline & $\begin{array}{r}\text { Elliptic } \\
(1999)\end{array}$ & $\begin{array}{l}\text { LOF } \\
(2000)\end{array}$ & $\begin{array}{c}\text { I-Forest } \\
(2008)\end{array}$ & $\begin{array}{l}\text { STA } \\
(2006)\end{array}$ & $\begin{array}{c}\text { MASTA } \\
(2015)\end{array}$ & $\begin{array}{r}\text { STenSr } \\
(2015)\end{array}$ & $\begin{array}{c}\text { Random Cut Forest } \\
\text { (2016) }\end{array}$ & $\begin{array}{l}\text { DenseAlert } \\
(2017)\end{array}$ & $\begin{array}{c}\text { MSTREAM } \\
(2021)\end{array}$ \\
\hline Group Anomalies & & & & & & & & $\sqrt{ }$ & $\checkmark$ \\
\hline Real-valued Features & $\checkmark$ & $\checkmark$ & $\checkmark$ & & & & $\checkmark$ & & $\boldsymbol{V}$ \\
\hline Constant Memory & & & & & & & $\checkmark$ & $\checkmark$ & $\boldsymbol{V}$ \\
\hline Const. Update Time & & & & $\checkmark$ & $\checkmark$ & $\checkmark$ & $\sqrt{ }$ & $\checkmark$ & $\mathcal{V}$ \\
\hline
\end{tabular}

learning based methods [69] [25]. However, we refrain from comparing with these approaches as they do not process the data in a streaming manner and typically require large amount of labelled training data, whereas we process the data in an unsupervised and online manner.

Note that Local Outlier Factor, Isolation Forest, Elliptic Envelope, STA, MASTA, STenSr, DenseAlert and Random Cut Forest are unsupervised algorithms. Of these, only DENSEALERT performs group anomaly detection (by detecting dense subtensors); however, as shown in Table 1, it cannot effectively handle real-valued features (as it treats all features as discrete-valued).

\section{PROBLEM}

Let $\mathcal{R}=\left\{r_{1}, r_{2}, \ldots\right\}$ be a stream of records, arriving in a streaming manner. Each record $r_{i}=\left(r_{i 1}, \ldots, r_{i d}\right)$ consists of $d$ attributes or dimensions, in which each dimension can either be categorical (e.g. IP address) or real-valued (e.g. average packet length). Note that since the data is arriving over time as a stream, we do not assume that the set of possible feature values is known beforehand; for example, in network traffic settings, it is common for new IP addresses to be seen for the first time at some point in the middle of the stream.

Our goal is to detect group anomalies. Intuitively, group anomalies should have the following properties:

(1) Similarity in Categorical Attributes: for categorical attributes, the group anomalies consist of a relatively small number of attribute values, repeated a suspiciously large number of times.

(2) Similarity in Real-Valued Attributes: for real-valued attributes, the group anomalies consist of clusters of numerically similar attribute values.

(3) Temporally Sudden: the group anomalies arrive suddenly, over a suspiciously short amount of time. In addition, their behavior (in terms of attribute values) should clearly differ from what we have observed previously, over the course of the stream.

\section{PROPOSED ALGORITHM}

\subsection{Motivation}

Consider the toy example in Table 2, comprising a stream of connections over time. This dataset shows a clear block of suspicious activity from time 4 to 5 , consisting of several IP addresses repeated a large number of times, as well as large packet sizes which seem to be anomalously large compared to the usual distribution of packet sizes.
Table 2: Simple toy example, consisting of a stream of multiaspect connections over time.

\begin{tabular}{ccccc}
\hline Time & Source IP & Dest. IP & Pkt. Size & $\cdots$ \\
\hline 1 & 194.027 .251 .021 & 194.027 .251 .021 & 100 & $\cdots$ \\
2 & 172.016 .113 .105 & 207.230 .054 .203 & 80 & $\cdots$ \\
4 & 194.027 .251 .021 & 192.168 .001 .001 & 1000 & $\cdots$ \\
4 & 194.027 .251 .021 & 192.168 .001 .001 & 995 & $\cdots$ \\
4 & 194.027 .251 .021 & 192.168 .001 .001 & 1000 & $\cdots$ \\
5 & 194.027 .251 .021 & 192.168 .001 .001 & 990 & $\cdots$ \\
5 & 194.027 .251 .021 & 194.027 .251 .021 & 1000 & $\cdots$ \\
5 & 194.027 .251 .021 & 194.027 .251 .021 & 995 & $\cdots$ \\
6 & 194.027 .251 .021 & 194.027 .251 .021 & 100 & $\cdots$ \\
7 & 172.016 .113 .105 & 207.230 .054 .203 & 80 & $\cdots$ \\
\hline
\end{tabular}

The main challenge, however, is to detect this type of patterns in a streaming manner, considering that we do not want to set any limits a priori on the duration of the anomalous activity we want to detect, or the number of IP addresses (or other attribute values) which may be involved in this activity.

As shown in Figure 1, our approach addresses these problems through the use of a number of locality-sensitive hash functions [9] which hash each incoming tuple into a fixed number of buckets. Intuitively, we do this such that tuples with many similar entries tend to be hashed into similar buckets. These hash functions are combined with a temporal scoring approach, which takes into account how much overlap we observe between the buckets at any time: high amounts of overlap arriving in a short period of time suggest the presence of anomalous activity.

In Sections 4.2 and 4.3, we describe our MSTREAM approach, and in Section 4.4, we describe our MSTREAM-PCA, MSTREAM-IB and MSTREAM-AE approaches which incorporate correlation between features in an unsupervised manner. MSTREAM-PCA uses principal component analysis, MSTREAM-IB uses information bottleneck, and MSTREAM-AE uses an autoencoder to first compress the original features and then apply MSTREAM in the compressed feature space.

\subsection{Hash Functions}

Our approach uses two types of hash functions: FeatureHash, which hashes each feature individually, and RECORDHAsH, which hashes an entire record jointly. We use multiple independent copies of each type of hash function, and explain how to combine these to produce a single anomalousness score. 


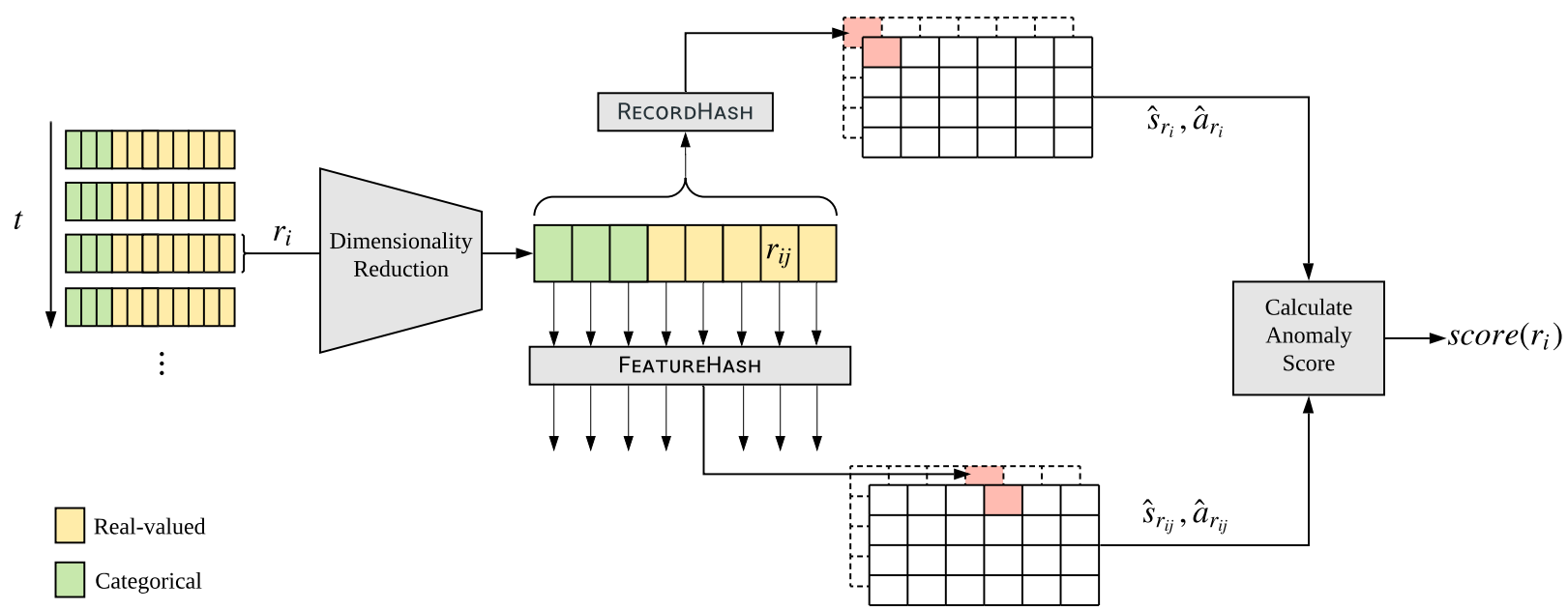

Figure 1: Diagram of the proposed MSTrEaM. The dimensionality reduction unit (Section 4.4) takes in a record and outputs a lower-dimensional embedding. Two types of locality-sensitive hash functions are then applied. FEATUREHASH (Algorithm 1) hashes each individual feature and RecordHash (Algorithm 2) hashes the entire record jointly. These are then combined together using a temporal scoring approach to calculate the anomaly score for the record (Algorithm 3).

4.2.1 FeatureHash. As shown in Algorithm 1, FeatureHash consists of hash functions independently applied to a single feature. There are two cases, corresponding to whether the feature is categorical (e.g. IP address) or real-valued (e.g. average packet length):

For categorical data, we use standard linear hash functions [31] which map integer-valued data randomly into $b$ buckets, i.e. $\{0, \ldots, b-1\}$, where $b$ is a fixed number.

For real-valued data, however, we find that randomized hash functions tend to lead to highly uneven bucket distributions for certain input datasets. Instead, we use a streaming log-bucketization approach. We first apply a log-transform to the data value (line 5), then perform min-max normalization, where the min and max are maintained in a streaming manner (line 7) and finally map it such that the range of feature values is evenly divided into $b$ buckets, i.e. $\{0, \ldots, b-1\}$ (line 8).

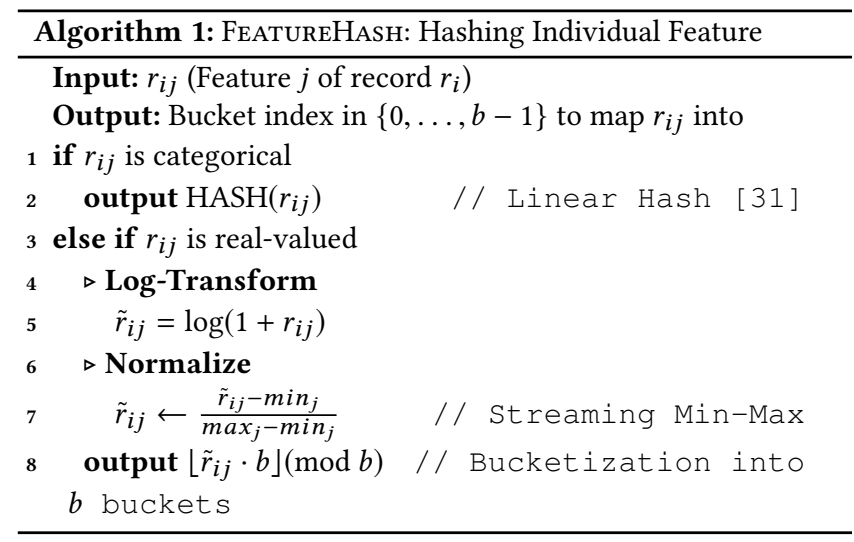

4.2.2 RecordHash. As shown in Algorithm 2, in RecordHash, we operate on all features of a record simultaneously. We first divide the entire record $r_{i}$ into two parts, one consisting of the categorical features $C$, say $r_{i}^{c a t}$, and the other consisting of realvalued features $\mathcal{R}$, say $r_{i}^{\text {num }}$. We then separately hash $r_{i}^{c a t}$ to get bucket $_{\text {cat }}$, and $r_{i}^{\text {num }}$ to get bucket $_{\text {num. }}$. Finally we take the sum modulo $b$ of bucket cat $_{\text {and }}$ and bet $t_{\text {num }}$ to get a bucket for $r_{i}$. We hash $r_{i}^{\text {cat }}$ and $r_{i}^{\text {num }}$ as follows:

(1) $r_{i}^{c a t}$ : We use standard linear hash functions [31] to map $\forall j \in C$ each of the individual features $r_{i j}$ into $b$ buckets, and then combine them by summing them modulo $b$ to compute the bucket index bucket $_{c a t}$ for $r_{i}^{\text {cat }}$ (line 3).

(2) $r_{i}^{\text {num }}$ : To compute the hash of a real-valued record $r_{i}^{\text {num }}$ of dimension $p=|\mathcal{R}|$, we choose $k$ random vectors $\mathbf{a}_{1}, \mathbf{a}_{2}, . ., \mathbf{a}_{\mathbf{k}}$ each having $p$ dimensions and independently sampled from a Gaussian distribution $\mathcal{N}_{p}\left(\mathbf{0}, \mathbf{I}_{\mathbf{p}}\right)$, where $k=\left\lceil\log _{2}(b)\right\rceil$. We compute the scalar product of $r_{i}^{\text {num }}$ with each of these vectors (line 6). We then map the positive scalar products to 1 and the non-positive scalar products to 0 and then concatenate these mapped values to get a $k$-bit string, then convert it from a bitset into an integer bucket $_{\text {num }}$ between 0 and $2^{k}-1$. (line 10).

\subsection{Temporal Scoring}

A recent algorithm, MIDAs [4], finds anomalous edges of a dynamic graph in a streaming manner. It combines a chi-squared goodnessof-fit test with the Count-Min-Sketch (CMS) [11] streaming data structures to get an anomaly score for each edge. In MidAs, $s_{u v}$ is defined to be the total number of edges from node $u$ to $v$ up to the current time $t$, while $a_{u v}$ is the number of edges from node $u$ to $v$ in the current time tick $t$ (but not including past time ticks). 


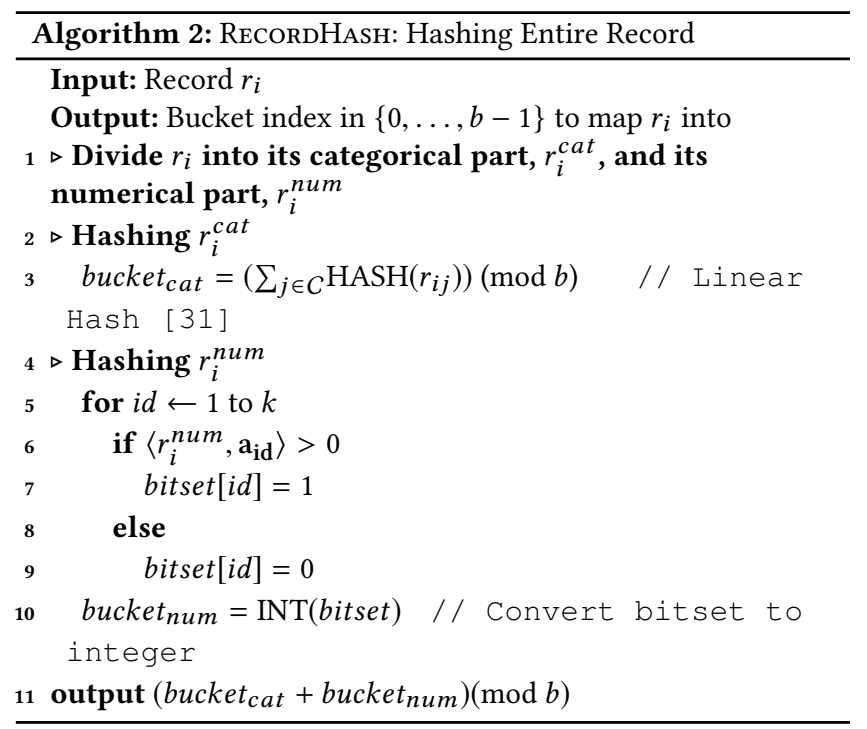

MidAs then divides the edges into two classes: edges at the current time tick $t\left(=a_{u v}\right)$, and edges in past time ticks $\left(=s_{u v}-a_{u v}\right)$, and computes the chi-squared statistic i.e. the sum over categories of $\frac{\text { (observed-expected })^{2}}{\text { expected }}$

$$
\begin{aligned}
& X^{2}=\frac{\left(\text { observed }_{(\text {cur_t } t)}-\operatorname{expected}_{\left(\text {cur_t }_{-}\right)}\right)^{2}}{\operatorname{expected}_{(\text {cur_t } t)}} \\
& +\frac{\left(\text { observed }_{(\text {past_t } t)}-\operatorname{expected}_{(\text {past_t }}\right)^{2}}{\text { expected }_{(\text {past_t } t)}} \\
& =\frac{\left(a_{u v}-\frac{s_{u v}}{t}\right)^{2}}{\frac{s_{u v}}{t}}+\frac{\left(\left(s_{u v}-a_{u v}\right)-\frac{t-1}{t} s_{u v}\right)^{2}}{\frac{t-1}{t} s_{u v}} \\
& =\left(a_{u v}-\frac{s_{u v}}{t}\right)^{2} \frac{t^{2}}{s_{u v}(t-1)}
\end{aligned}
$$

MIDAs uses two types of CMS data structures to maintain approximate counts $\hat{s}_{u v}$ and $\hat{a}_{u v}$ which estimate $s_{u v}$ and $a_{u v}$ respectively. The anomaly score for an edge in MIDAs is then defined as:

$$
\operatorname{score}(u, v, t)=\left(\hat{a}_{u v}-\frac{\hat{s}_{u v}}{t}\right)^{2} \frac{t^{2}}{\hat{s}_{u v}(t-1)}
$$

However, Midas is designed to detect anomalous edges, which are two-dimensional records (consisting of source and destination node index). Therefore, it cannot be applied in the high-dimensional setting of multi-aspect data. Moreover, MidAs treats variables of the dataset as categorical variables, whereas multi-aspect data can contain arbitrary mixtures of categorical variables (e.g. source IP address) and numerical variables (e.g. average packet size).

We extend Midas to define an anomalousness score for each record and detect anomalous records in a streaming manner. Given each incoming record $r_{i}$ having $j$ features, we can compute $j+1$ anomalousness scores: one for the entire record $r_{i}$ and one for each individual feature $r_{i j}$. We compute each score by computing the chi-squared statistic over the two categories: current time tick and past time ticks. Anomaly scores for individual attributes are useful for interpretability, as they help explain which features are most responsible for the anomalousness of the record. Finally, we combine these scores by taking their sum.

Definition 1 (ANOMAly ScoRe). Given a newly arriving record $\left(r_{i}, t\right)$, our anomalousness score is computed as:

$$
\operatorname{score}\left(r_{i}, t\right)=\left(\hat{a}_{r_{i}}-\frac{\hat{s}_{r_{i}}}{t}\right)^{2} \frac{t^{2}}{\hat{s}_{r_{i}}(t-1)}+\sum_{j=1}^{d} \operatorname{score}\left(r_{i j}, t\right)
$$

where,

$$
\operatorname{score}\left(r_{i j}, t\right)=\left(\hat{a}_{r_{i j}}-\frac{\hat{s}_{r_{i j}}}{t}\right)^{2} \frac{t^{2}}{\hat{s}_{r_{i j}}(t-1)}
$$

and $\hat{a}_{r_{i}}\left(\right.$ or $\left.\hat{a}_{r_{i j}}\right)$ is an approximate count of $r_{i}\left(\right.$ or $\left.r_{i j}\right)$ at current time $t$ and $\hat{s}_{r_{i}}\left(\right.$ or $\left.\hat{s}_{r_{i j}}\right)$ is an approximate count of $r_{i}$ (or $\left.r_{i j}\right)$ up to time $t$.

We also allow temporal flexibility of records, i.e. records in the recent past count towards the current anomalousness score. This is achieved by reducing the counts $\hat{a}_{r_{i}}$ and $\hat{a}_{r_{i j}} \forall j \in\{1, \ldots, d\}$ by a factor of $\alpha \in(0,1)$ rather than resetting them at the end of each time tick. This results in past records counting towards the current time tick, with a diminishing weight.

MSTREAM is summarised in Algorithm 3.

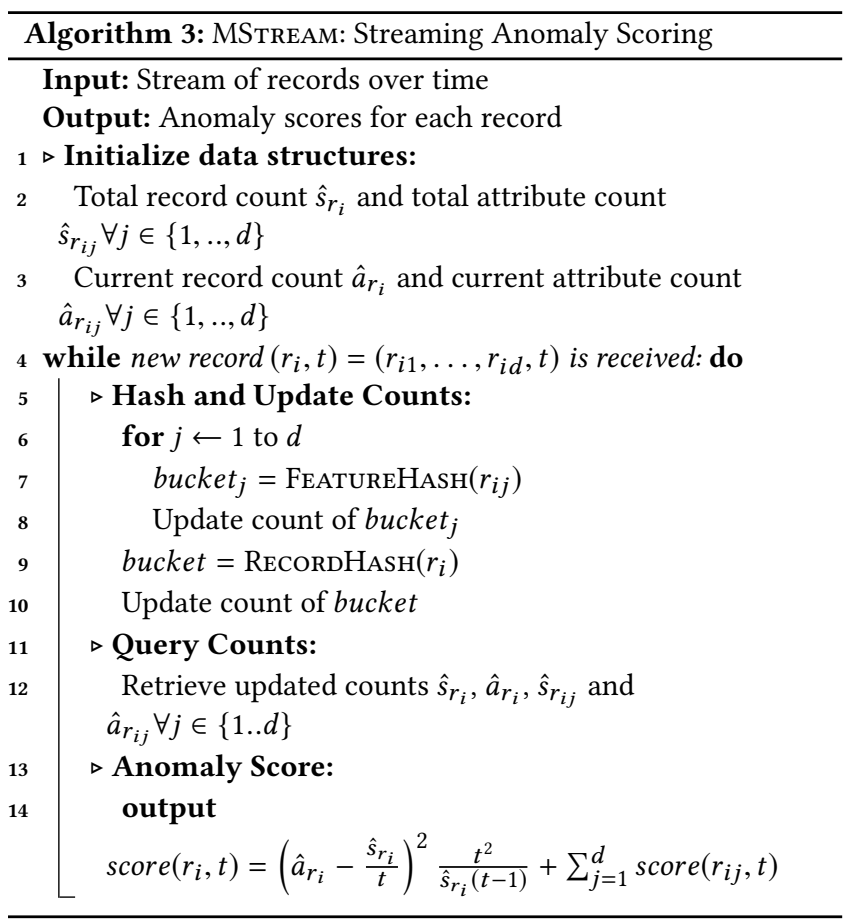

\subsection{Incorporating Correlation Between Features}

In this section, we describe our MSTREAM-PCA, MSTREAM-IB and MSTREAM-AE approaches where we run the MSTREAM algorithm on 
a lower-dimensional embedding of the original data obtained using Principal Component Analysis (PCA) [47], Information Bottleneck (IB) [66] and Autoencoder (AE) [22] methods in a streaming manner.

Our motivation for combining PCA, IB and AE methods with MSTREAm is two-fold. Firstly, the low-dimensional representations learned by these algorithms incorporate correlation between different attributes of the record, making anomaly detection more effective. Secondly, a reduction in the dimensions would result in faster processing per record.

For all three methods, we first learn the dimensionality reduction transformation using a very small initial subset of 256 records from the incoming stream. We then compute the embeddings for the subsequent records and pass them to MSTREAM to detect anomalies in an online manner.

Principal Component Analysis. We choose PCA because it only requires one major parameter to tune: namely the dimension of the projection space. Moreover, this parameter can be set easily by analysis of the explained variance ratios of the principal components. Hence MSTREAM-PCA can be used as an off-the-shelf algorithm for streaming anomaly detection with dimensionality reduction.

Information Bottleneck. Information bottleneck for dimensionality reduction can be posed as the following optimization problem:

$$
\min _{p(t \mid x)} I(X ; T)-\beta I(T ; Y)
$$

where $X, Y$, and $T$ are random variables. $T$ is the compressed representation of $X, I(X ; T)$ and $I(T ; Y)$ are the mutual information of $X$ and $T$, and of $T$ and $Y$, respectively, and $\beta$ is a Lagrange multiplier In our setting, $X$ denotes the multi-aspect data, $Y$ denotes whether the data is anomalous and $T$ denotes the dimensionally reduced features that we wish to find. Our implementation is based on the Neural Network approach for Nonlinear Information Bottleneck [26].

Autoencoder. Autoencoder is a neural network based approach for dimensionality reduction. An autoencoder network consists of an encoder and a decoder. The encoder compresses the input into a lower-dimensional space, while the decoder reconstructs the input from the low-dimensional representation. Our experimental results in Section 5 show that even with a simple 3 layered autoencoder, MSTREAM-AE outperforms both MSTREAM-PCA and MSTREAM-IB.

\subsection{Time and Memory Complexity}

In terms of memory, MSTREAM only needs to maintain data structures over time, which requires memory proportional to $O(w b d)$, where $w, b$ and $d$ are the number of hash functions, the number of buckets in the data structures and the total number of dimensions; which is bounded with respect to the stream size.

For time complexity, the only relevant steps in Algorithm 3 are those that either update or query the data structures, which take $O(w d)$ (all other operations run in constant time). Thus, the time complexity per update step is $O(w d)$.

\section{EXPERIMENTS}

In this section, we evaluate the performance of MSTREAM and MStreAm-AE compared to Elliptic Envelope, LOF, I-Forest, Random Cut Forest and DENSEALERT on multi-aspect data streams. We aim to answer the following questions:

Q1. Anomaly Detection Performance: How accurately does MSTREAM detect real-world anomalies compared to baselines, as evaluated using the ground truth labels?

Q2. Scalability: How does it scale with input stream length and number of dimensions? How does the time needed to process each input compare to baseline approaches?

Q3. Real-World Effectiveness: Does it detect meaningful anomalies? Does it detect group anomalies?

Datasets. KDDCUP99 dataset [12] is based on the DARPA dataset and is among the most extensively used datasets for intrusion detection. Since the proportion of data belonging to the 'attack' class is much larger than the proportion of data belonging to the 'non-attack' class, we downsample the 'attack' class to a proportion of $20 \%$. KDDCUP99 has 42 dimensions and 1.21 million records.

[55] surveys different intrusion detection datasets and recommends to use the newer CICIDS [59] and UNSW-NB15 [39] datasets. These contain modern-day attacks and follow the established guidelines for reliable intrusion detection datasets (in terms of realism, evaluation capabilities, total capture, completeness, and malicious activity) [59].

CICIDS 2018 dataset was generated at the Canadian Institute of Cybersecurity. Each record is a flow containing features such as Source IP Address, Source Port, Destination IP Address, Bytes, and Packets. These flows were captured from a real-time simulation of normal network traffic and synthetic attack simulators. This consists of the CICIDS-DoS dataset (1.05 million records, 80 features) and the CICIDS-DDoS dataset (7.9 million records, 83 features). CICIDS-DoS has $5 \%$ anomalies whereas CICIDS-DDoS has $7 \%$ anomalies.

UNSW-NB 15 dataset was created by the Cyber Range Lab of the Australian Centre for Cyber Security (ACCS) for generating a hybrid of real modern normal activities and synthetic contemporary attack behaviors. This dataset has nine types of attacks, namely, Fuzzers, Analysis, Backdoors, DoS, Exploits, Generic, Reconnaissance, Shellcode and Worms. It has 49 features and 2.5 million records including $13 \%$ anomalies.

Baselines. As described in Section 2, we are in the streaming unsupervised learning regime, and hence do not compare with supervised or offline algorithms.

We consider Elliptic Envelope, Local Outlier Factor, Isolation Forest, STA, MASTA, STenSr, DenseAlert and Random Cut Forest since they operate on multi-aspect data, however, due to a large number of dimensions, even sparse tensor versions of STA/MASTA/STenSr run out of memory on these datasets. So, we compare with Elliptic Envelope, Local Outlier Factor, Isolation Forest, DenseAlert and Random Cut Forest.

Evaluation Metrics. All the methods output an anomaly score per edge (higher is more anomalous). We plot the ROC curve, which compares the True Positive Rate (TPR) and False Positive Rate (FPR), 
Table 3: AUC of each method on different datasets.

\begin{tabular}{rccccccccc}
\hline & Elliptic & LOF & I-Forest & DAlert & RCF & MSTREAM & MSTREAM-PCA & MSTREAM-IB & MSTREAM-AE \\
\hline KDD & $0.34 \pm 0.025$ & 0.34 & $0.81 \pm 0.018$ & 0.92 & 0.63 & $0.91 \pm 0.016$ & $0.92 \pm 0.000$ & $\mathbf{0 . 9 6} \pm 0.002$ & $\mathbf{0 . 9 6} \pm 0.005$ \\
DoS & $0.75 \pm 0.021$ & 0.50 & $0.73 \pm 0.008$ & 0.61 & 0.83 & $0.93 \pm 0.001$ & $0.92 \pm 0.001$ & $\mathbf{0 . 9 5} \pm 0.003$ & $0.94 \pm 0.001$ \\
UNSW & $0.25 \pm 0.003$ & 0.49 & $0.84 \pm 0.023$ & 0.80 & 0.45 & $0.86 \pm 0.001$ & $0.81 \pm 0.001$ & $0.82 \pm 0.001$ & $\mathbf{0 . 9 0} \pm 0.001$ \\
DDoS & $0.57 \pm 0.106$ & 0.46 & $0.56 \pm 0.021$ & -- & 0.63 & $0.91 \pm 0.000$ & $\mathbf{0 . 9 4} \pm 0.000$ & $0.82 \pm 0.000$ & $0.93 \pm 0.000$ \\
\hline
\end{tabular}

without needing to fix any threshold. We also report the ROC-AUC (Area under the ROC curve).

Experimental Setup. All experiments are carried out on a 2.4GHz Intel Core $i 9$ processor, 32GB RAM, running OS $X$ 10.15.2. We implement MSTREAM in $\mathrm{C}++$. We use 2 independent copies of each hash function, and we set the number of buckets to 1024 . We set the temporal decay factor $\alpha$ as 0.85 for KDDCUP99, 0.95 for CICIDS-DoS and CICIDS-DDoS, and 0.4 for UNSW-NB 15 due to its higher time granularity. Note that MSTREAM is not sensitive to variation of $\alpha$ parameter as shown in Table 5. Since KDDCUP99 dataset does not have timestamps, we apply the temporal decay factor once every 1000 records. We discuss the influence of temporal decay factor $\alpha$ on the ROC-AUC in Appendix A.

To demonstrate the robustness of our proposed approach, we set the output dimension of MSTREAM-PCA, MSTREAM-IB and MSTREAM-AE for all datasets to a common value of 12 instead of searching individually on each method and dataset. We reduce the real-valued columns to 12 dimensions and then pass these along with the categorical columns to MSTREAM. Results on varying the number of output dimensions can be found in the Appendix. For MSTREAM-PCA we use the open-source implementation of PCA available in the scikit-learn [48] library. Parameters for MSTREAM$\mathrm{AE}$ and MSTREAM-IB are described in Appendix C.

We use open-sourced implementations of DENSEALERT and Random Cut Forest, provided by the authors, following parameter settings as suggested in the original papers. For Elliptic Envelope, Local Outlier Factor and Isolation Forest we use the open-source implementation available in the scikit-learn [48] library. We also pass the true anomaly percentage to Elliptic Envelope, Local Outlier Factor and Isolation Forest methods, while the remainder of the methods do not require the anomaly percentage.

All the experiments, unless explicitly specified, are performed 5 times for each parameter group, and the mean and standard deviation values are reported.

\subsection{Anomaly Detection Performance}

Figure 2 plots the ROC curve for MSTREAM, MSTREAM-PCA, MSTREAM-IB and MSTREAM-AE along with the baselines, Elliptic Envelope, Local Outlier Factor, Isolation Forest, DenseAlert and Random Cut Forest on CICIDS-DoS dataset. We see that MSTrEam, MSTREAM-PCA, MSTREAM-IB and MSTREAM-AE achieve a much higher ROC-AUC $(0.92-0.95)$ compared to the baselines. MSTREAM and its variants achieve at least 50\% higher AUC than DenseAlert, $11 \%$ higher than Random Cut Forest 26\% higher than Isolation Forest, $23 \%$ higher than Elliptic Envelope and $84 \%$ higher than Local Outlier Factor.

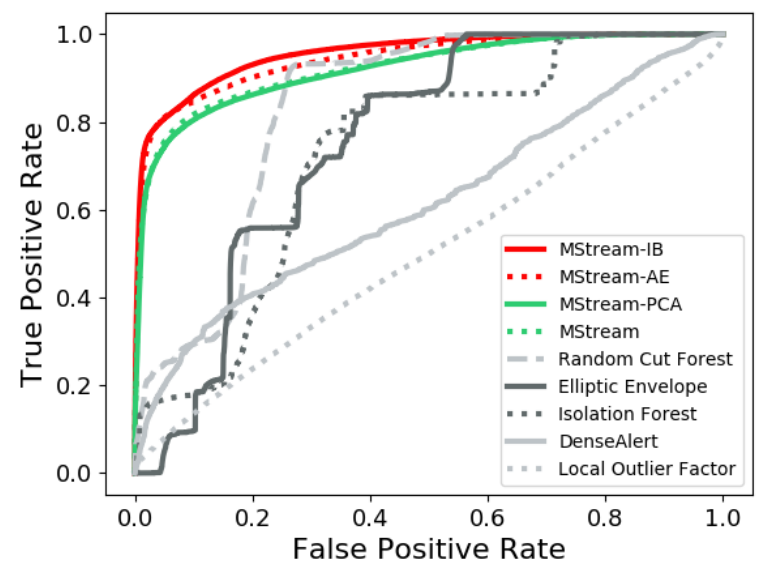

Figure 2: ROC on CICIDS-DoS dataset.

Table 3 shows the AUC of Elliptic Envelope, Local Outlier Factor, Isolation Forest, DENSEALERT, Random Cut Forest and MSTREAM on KDDCUP99, CICIDS-DoS, UNSW-NB 15 and CICIDS-DDoS datasets. We report a single value for Local Outlier Factor and DenseALERT because these are non-randomized methods. We also report a single value for Random Cut Forest because we use the parameters and random seed of the original implementation. DENSEALERT performs well on small sized datasets such as KDDCUP99 but as the dimensions increase, its performance decreases. On the large CICIDS-DDoS dataset DENSEALERT runs out of memory. We observe that MSTREAM outperforms all baselines on all datasets. By learning the correlation between features, MSTREAM-AE achieves higher ROC-AUC than MSTREAM, and performs comparably or better than MSTREAM-PCA and MSTREAM-IB. We also discuss evaluating the ROC-AUC in a streaming manner in Appendix D.

Figure 3 plots ROC-AUC vs. running time (log-scale, in seconds, excluding $\mathrm{I} / \mathrm{O}$ ) for the different methods on the CICIDS-DoS dataset. We see that MSTREAM, MSTREAM-PCA, MSTREAM-IB and MSTREAMAE achieve $11 \%$ to $90 \%$ higher AUC compared to baselines, while also running almost two orders of magnitude faster.

\subsection{Scalability}

Table 4 shows the time it takes Elliptic Envelope, Local Outlier Factor, Isolation Forest, DenseAlert, Random Cut Forest, MStream and MSTREAM-AE to run on KDDCUP99, CICIDS-DoS, UNSW-NB 15 and CICIDS-DDoS datasets. We see that MSTREAM runs much faster than the baselines: for example, MSTREAm is 79 times faster than 
Table 4: Running time of each method on different datasets in seconds.

\begin{tabular}{rccccccccc}
\hline & Elliptic & LOF & I-Forest & DAlert & RCF & MStream & MSTream-PCA & MStream-IB & MSTream-AE \\
\hline KDD & 216.3 & 1478.8 & 230.4 & 341.8 & 181.6 & 4.3 & 2.5 & 3.1 & 3.1 \\
DoS & 455.8 & 398.8 & 384.8 & 333.4 & 459.4 & 10.4 & 2.1 & 3.7 & 5.1 \\
UNSW & 654.6 & 2091.1 & 627.4 & 329.6 & 683.8 & 12.8 & 6.6 & 8 & 8 \\
DDoS & 3371.4 & $15577 s$ & 3295.8 & -- & 4168.8 & 61.6 & 16.9 & 25.6 & 27.7 \\
\hline
\end{tabular}

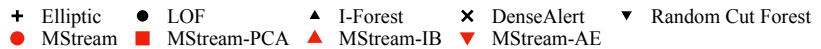

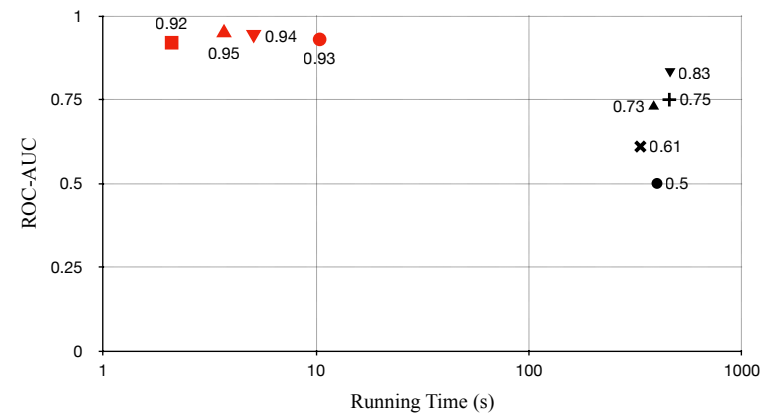

Figure 3: ROC-AUC vs time on CICIDS-DoS dataset.

DenseAlert on the KDDCUP99 dataset. MSTREAM-PCA, MSTREAMIB and MSTREAM-AE incorporate dimensionality reduction and are therefore faster than MSTREAM: for example, MSTREAM-AE is 1.38 times faster than MSTREAm and 110 times faster than DENSEALERT on the KDDCUP99 dataset.

Figure 4 shows the scalability of MSTREAM with respect to the number of records in the stream (log-scale). We plot the time needed to run on the (chronologically) first $2^{12}, 2^{13}, 2^{14}, \ldots, 2^{20}$ records of the CICIDS-DoS dataset. Each record has 80 dimensions. This confirms the linear scalability of MSTREAM with respect to the number of records in the input stream due to its constant processing time per record.

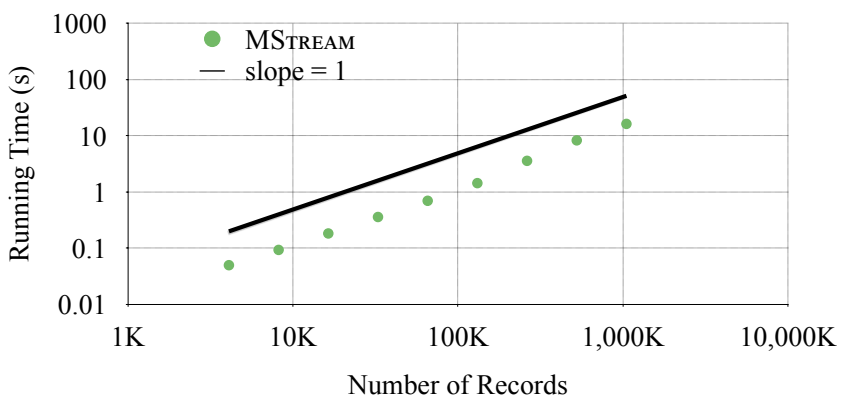

Figure 4: MSTREAM scales linearly with the number of records in CICIDS-DoS.

Figure 5 shows the scalability of MSTREAM with respect to the number of dimensions (linear-scale). We plot the time needed to run on the first $10,20,30, \ldots, 80$ dimensions of the CICIDS-DoS dataset.
This confirms the linear scalability of MSTREAM with respect to the number of dimensions in the input data.

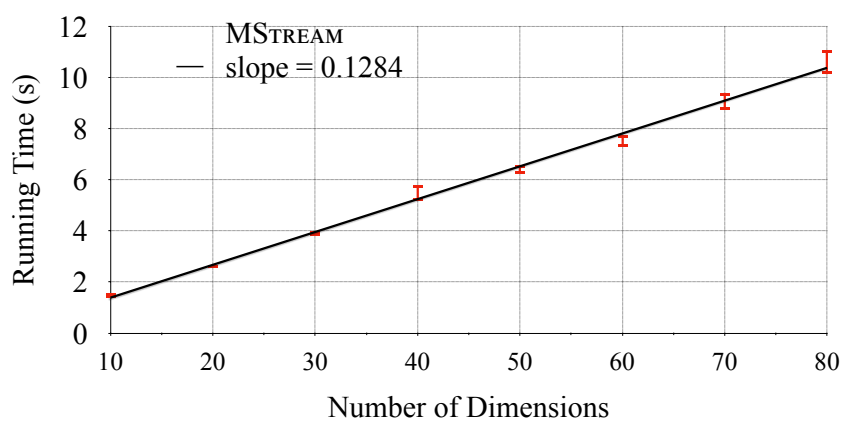

Figure 5: MSTREAM scales linearly with the number of dimensions in CICIDS-DoS.

Figure 6 shows the scalability of MSTREAM with respect to the number of hash functions (linear-scale). We plot the time taken to run on the CICIDS-DoS dataset with 2,3, 4 hash functions. This confirms the linear scalability of MSTREAM with respect to the number of hash functions.

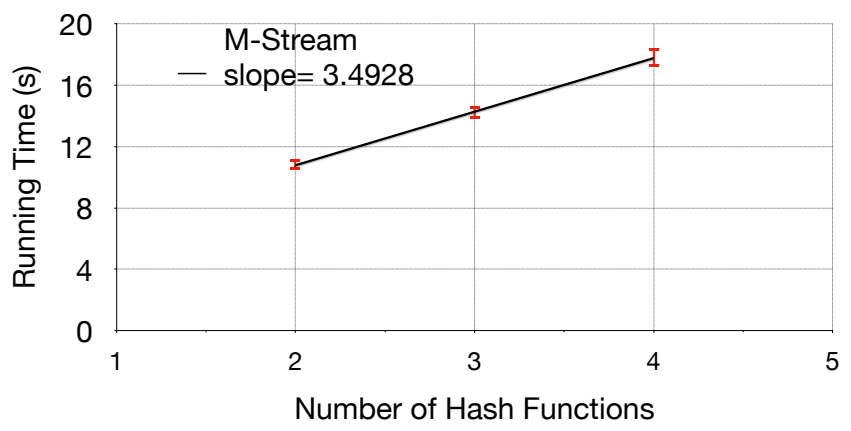

Figure 6: MSTREAM scales linearly with the number of hash functions in CICIDS-DoS.

Since MStream-PCA, MStream-IB and MStream-AE apply MSTREAM on the lower-dimensional features obtained using an autoencoder, they are also scalable.

Figure 7 plots a frequency distribution of the time taken (in microseconds) to process each record in the CICIDS-DoS dataset. MSTREAM processes $957 \mathrm{~K}$ records within $10 \mu \mathrm{s}$ each, $60 \mathrm{~K}$ records within $100 \mu$ s each and remaining $30 \mathrm{~K}$ records within $1000 \mu$ s each. 


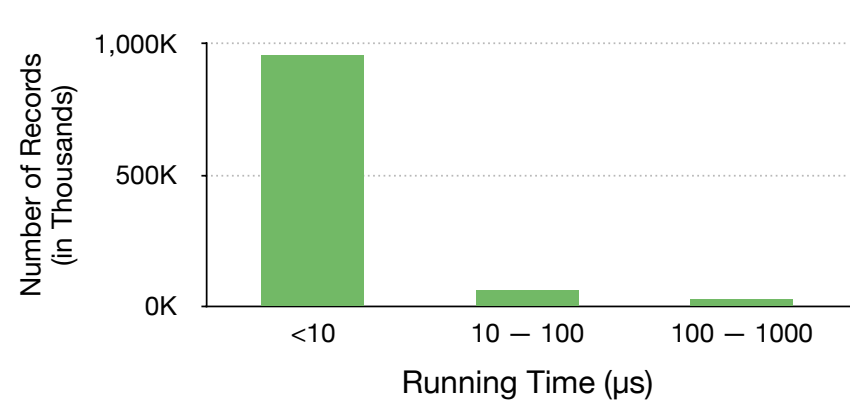

Figure 7: Distribution of processing times for $\sim 1.05 \mathrm{M}$ records of the CICIDS-DoS dataset.

\subsection{Discoveries}

We plot normalized anomaly scores over time using Elliptic Envelope, Local Outlier Factor, Isolation Forest, DenseAlert, Random Cut Forest and MSTrEAm on the CICIDS-DoS dataset in Figure 8. To visualize, we aggregate records occurring in each minute by taking the max anomaly score per minute, for a total of 565 minutes. Ground truth values are indicated by points plotted at $y=0$ (i.e. normal) or $y=1$ (anomaly).

Local Outlier Factor and DenseAlert miss many anomalies whereas Elliptic Envelope, Isolation Forest and Random Cut Forest output many high scores unrelated to any attacks. This is also reflected in Table 3 and shows that MSTREAM is effective in catching real-world anomalies.

Group anomaly detection: In Figure $8, G$ is a group anomaly which MSTREAm is able to detect, whereas Elliptic Envelope, Local Outlier Factor and Isolation Forest completely miss it. DENSEALERT and Random Cut Forest partially catch it, but are also not fully effective in such high-dimensional datasets. This shows the effectiveness of MSTREAM in catching group anomalies such as DoS and DDoS attacks.

Explainability: As MSTREAM estimates feature-specific anomaly scores before aggregating them, it is interpretable. For a given anomaly, we can rank the features according to their anomaly scores. We can then explain which features were most responsible for the anomalousness of a record in an unsupervised setting.

For example, in Figure 8, MSTream finds that $e$ is an anomaly that occurs due to the Flow IAT Min feature. This agrees with [59], which finds that the best feature set for DoS using a Random Forest approach (supervised learning; in contrast, our approach does not require labels) are B.Packet Len Std, Flow IAT Min, Fwd IAT Min, and Flow IAT Mean.

\section{CONCLUSION}

In this paper, we proposed MSTREAM for detecting group anomalies in multi-aspect streams, and MSTREAM-PCA, MSTREAM-IB and MSTREAM-AE which incorporate dimensionality reduction to improve accuracy and speed. Future work could consider more complex combinations (e.g. weighted sums) of anomaly scores for individual attributes. Our contributions are:

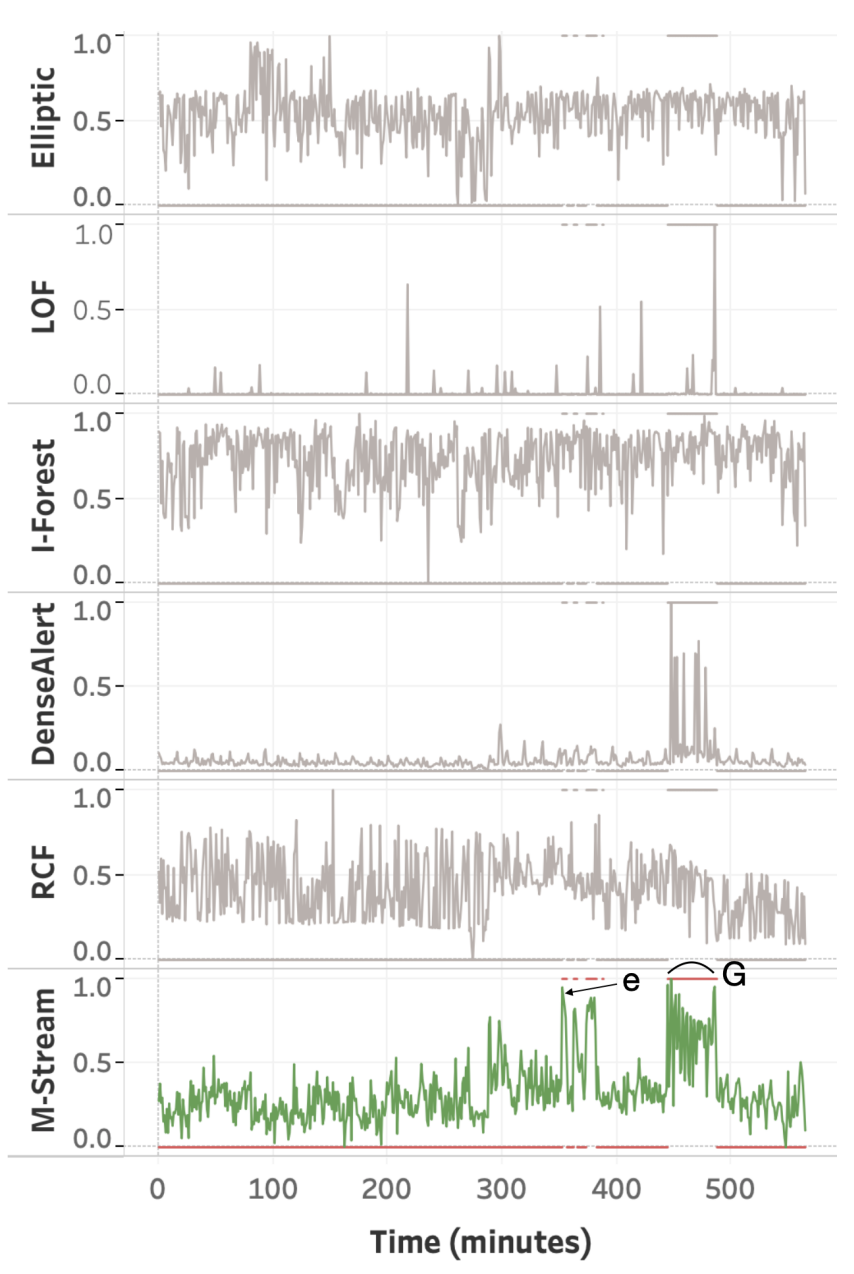

Figure 8: Plots of anomaly scores over time; spikes for MSTREAM correspond to the ground truth events in CICIDSDoS, but not for baselines.

(1) Multi-Aspect Group Anomaly Detection: We propose a novel approach for detecting group anomalies in multiaspect data, including both categorical and numeric attributes.

(2) Streaming Approach: Our approach processes the data in a fast and streaming fashion, performing each update in constant time and memory.

(3) Effectiveness: Our experimental results show that MSTREAM outperforms baseline approaches.

\section{ACKNOWLEDGMENTS}

This work was supported in part by NUS ODPRT Grant R-252-000A81-133. The authors would like to thank Thijs Laarhoven for his suggestions.

\section{REFERENCES}

[1] Leman Akoglu, Hanghang Tong, and Danai Koutra. 2015. Graph Based Anomaly Detection and Description: A Survey. Data mining and knowledge discovery (2015). 
[2] Azeem Aqil, Karim Khalil, Ahmed O F Atya, Evangelos E Papalexakis, Srikanth V Krishnamurthy, Trent Jaeger, K K Ramakrishnan, Paul Yu, and Ananthram Swami. 2017. Jaal: Towards Network Intrusion Detection at ISP Scale. In CoNEXT.

[3] Elisa Bertino, Evimaria Terzi, Ashish Kamra, and Athena Vakali. 2005. Intrusion detection in RBAC-administered databases. In ACSAC.

[4] Siddharth Bhatia, Bryan Hooi, Minji Yoon, Kijung Shin, and Christos Faloutsos. 2020. MIDAS: Microcluster-Based Detector of Anomalies in Edge Streams. In AAAI.

[5] Petko Bogdanov, Christos Faloutsos, Misael Mongiovì, Evangelos E Papalexakis, Razvan Ranca, and Ambuj K Singh. 2013. NetSpot: Spotting Significant Anomalous Regions on Dynamic Networks. In $S D M$.

[6] Francesco Bonchi, Ilaria Bordino, Francesco Gullo, and Giovanni Stilo. 2016 Identifying Buzzing Stories via Anomalous Temporal Subgraph Discovery. In WI.

[7] Francesco Bonchi, Ilaria Bordino, Francesco Gullo, and Giovanni Stilo. 2019. The importance of unexpectedness: Discovering buzzing stories in anomalous temporal graphs. Web Intelligence (2019).

[8] Markus M Breunig, Hans-Peter Kriegel, Raymond T Ng, and Jörg Sander. 2000 LOF: identifying density-based local outliers. In SIGMOD.

[9] Moses S Charikar. 2002. Similarity estimation techniques from rounding algorithms. In STOC.

[10] L Chi, B Li, X Zhu, S Pan, and L Chen. 2018. Chang, Yen-Yu and Li, Pan and Sosic, Rok and Afifi, MH and Schweighauser, Marco and Leskovec, Jure. IEEE Transactions on Cybernetics (2018).

[11] Graham Cormode and Shan Muthukrishnan. 2005. An improved data stream summary: the count-min sketch and its applications. Fournal of Algorithms (2005).

[12] KDD Cup 1999 Dataset. 1999. http://kdd.ics.uci.edu/databases/kddcup99/ kddcup99.html.

[13] Paulo Vitor de Campos Souza, Augusto Junio Guimarães, Thiago Silva Rezende Vinicius Jonathan Silva Araujo, and Vanessa Souza Araujo. 2020. Detection of Anomalies in Large-Scale Cyberattacks Using Fuzzy Neural Networks. Artificial Intelligence (2020).

[14] Dhivya Eswaran and Christos Faloutsos. 2018. Sedanspot: Detecting anomalies in edge streams. In ICDM.

[15] Hadi Fanaee-T and João Gama. 2015. Multi-aspect-streaming tensor analysis. Knowledge-Based Systems (2015).

[16] Hadi Fanaee-T and João Gama. 2016. Tensor-based anomaly detection: An interdisciplinary survey. Knowledge-Based Systems (2016).

[17] Adam Goodge, Bryan Hooi, See-Kiong Ng, and Wee Siong Ng. 2020. Robustness of Autoencoders for Anomaly Detection Under Adversarial Impact. In IfCAI.

[18] Tyrone Gradison and Evimaria Terzi. 2018. Intrusion Detection Technology. In Encyclopedia of Database Systems.

[19] Sudipto Guha, Nina Mishra, Gourav Roy, and Okke Schrijvers. 2016. Robust Random Cut Forest Based Anomaly Detection on Streams. In ICML.

[20] Nikhil Gupta, Dhivya Eswaran, Neil Shah, Leman Akoglu, and Christos Faloutsos. 2017. LookOut on Time-Evolving Graphs: Succinctly Explaining Anomalies from Any Detector. ArXiv abs/1710.05333 (2017).

[21] Kawther Hassine, Aiman Erbad, and Ridha Hamila. 2019. Important Complexity Reduction of Random Forest in Multi-Classification Problem. In IWCMC.

[22] Geoffrey E Hinton and Richard S Zemel. 1994. Autoencoders, minimum description length and Helmholtz free energy. In NIPS.

[23] Meng Jiang, Alex Beutel, Peng Cui, Bryan Hooi, Shiqiang Yang, and Christos Faloutsos. 2015. A general suspiciousness metric for dense blocks in multimodal data. In ICDM.

[24] Hyunjun Ju, Dongha Lee, Junyoung Hwang, Junghyun Namkung, and Hwanjo Yu 2020. PUMAD: PU Metric learning for anomaly detection. Information Sciences (2020).

[25] Farrukh Aslam Khan, Abdu Gumaei, Abdelouahid Derhab, and Amir Hussain 2019. A Novel Two-Stage Deep Learning Model for Efficient Network Intrusion Detection. IEEE Access (2019).

[26] Artemy Kolchinsky, Brendan D Tracey, and David H Wolpert. 2019. Nonlinear Information Bottleneck. Entropy (2019).

[27] Tamara G Kolda and Brett W Bader. 2009. Tensor decompositions and applications. SIAM review (2009).

[28] Xiangnan Kong and S Yu Philip. 2011. An ensemble-based approach to fast classification of multi-label data streams. In CollaborateCom.

[29] Rithesh Kumar, Anirudh Goyal, Aaron C Courville, and Yoshua Bengio. 2019 Maximum Entropy Generators for Energy-Based Models. ArXiv abs/1901.08508 (2019).

[30] Jie Li, Guan Han, Jing Wen, and Xinbo Gao. 2011. Robust tensor subspace learning for anomaly detection. IfMLC (2011).

[31] Witold Litwin. 1980. Linear hashing: a new tool for file and table addressing.. In $V L D B$.

[32] Fei Tony Liu, Kai Ming Ting, and Zhi-Hua Zhou. 2008. Isolation Forest. ICDM (2008).

[33] Chen Luo and Anshumali Shrivastava. 2018. Arrays of (Locality-Sensitive) Count Estimators (ACE): Anomaly Detection on the Edge. In $W W W$.
[34] Fragkiskos D Malliaros, Vasileios Megalooikonomou, and Christos Faloutsos. 2012. Fast Robustness Estimation in Large Social Graphs: Communities and Anomaly Detection. In SDM.

[35] Emaad A Manzoor, Hemank Lamba, and Leman Akoglu. 2018. xStream: Outlier Detection in Feature-Evolving Data Streams. In KDD.

[36] Hing-Hao Mao, Chung-Jung Wu, Evangelos E Papalexakis, Christos Faloutsos, Kuo-Chen Lee, and Tien-Cheu Kao. 2014. MalSpot: Multi 2 malicious network behavior patterns analysis. In PAKDD.

[37] Koji Maruhashi, Fan Guo, and Christos Faloutsos. 2011. Multiaspectforensics: Pattern mining on large-scale heterogeneous networks with tensor analysis. In ASONAM.

[38] Misael Mongiovì, Petko Bogdanov, Razvan Ranca, Ambuj K Singh, Evangelos E Papalexakis, and Christos Faloutsos. 2012. SigSpot: Mining Significant Anomalous Regions from Time-Evolving Networks (Abstract Only). In SIGMOD.

[39] Nour Moustafa and Jill Slay. 2015. UNSW-NB15: a comprehensive data set for network intrusion detection systems (UNSW-NB15 network data set). In MilCIS.

[40] Gyoung S Na, Donghyun Kim, and Hwanjo Yu. 2018. DILOF: Effective and Memory Efficient Local Outlier Detection in Data Streams. In KDD.

[41] Phuc Cuong Ngo, Amadeus Aristo Winarto, Connie Khor Li Kou, Sojeong Park, Farhan Akram, and Hwee Kuan Lee. 2019. Fence GAN: Towards Better Anomaly Detection. ICTAI (2019).

[42] Shirui Pan, Jia Wu, Xingquan Zhu, and Chengqi Zhang. 2015. Graph Ensemble Boosting for Imbalanced Noisy Graph Stream Classification. IEEE Transactions on Cybernetics (2015).

[43] Shirui Pan, Kuan Wu, Yang Zhang, and Xue Li. 2010. Classifier Ensemble for Uncertain Data Stream Classification. In Advances in Knowledge Discovery and Data Mining.

[44] Shirui Pan, Xingquan Zhu, Chengqi Zhang, and S Yu Philip. 2013. Graph stream classification using labeled and unlabeled graphs. In ICDE

[45] Evangelos Papalexakis, Konstantinos Pelechrinis, and Christos Faloutsos. 2014. Spotting misbehaviors in location-based social networks using tensors. In $W W W$.

[46] Evangelos E Papalexakis, Christos Faloutsos, and Nicholas D Sidiropoulos. 2012. Parcube: Sparse parallelizable tensor decompositions. In ECMLPKDD.

[47] Karl Pearson. 1901. LIII. On lines and planes of closest fit to systems of points in space. The London, Edinburgh, and Dublin Philosophical Magazine and fournal of Science (1901).

[48] Fabian Pedregosa, Gaël Varoquaux, Alexandre Gramfort, Vincent Michel, Bertrand Thirion, Olivier Grisel, Mathieu Blondel, Peter Prettenhofer, Ron Weiss, Vincent Dubourg, et al. 2011. Scikit-learn: Machine Learning in Python. FMLR (2011).

[49] Bryan Perozzi and Leman Akoglu. 2016. Scalable anomaly ranking of attributed neighborhoods. In SDM.

[50] Bryan Perozzi and Leman Akoglu. 2018. Discovering Communities and Anomalies in Attributed Graphs: Interactive Visual Exploration and Summarization. TKDD (2018).

[51] Bryan Perozzi, Michael Schueppert, Jack Saalweachter, and Mayur Thakur. 2016. When Recommendation Goes Wrong: Anomalous Link Discovery in Recommendation Networks. In $K D D$.

[52] Smitha Rajagopal, Katiganere Siddaramappa Hareesha, and Poornima Panduranga Kundapur. 2020. Feature Relevance Analysis and Feature Reduction of UNSW NB-15 Using Neural Networks on MAMLS. In ICACIE.

[53] Smitha Rajagopal, Poornima Panduranga Kundapur, and Katiganere Siddaramappa Hareesha. 2020. A Stacking Ensemble for Network Intrusion Detection Using Heterogeneous Datasets. Security and Communication Networks (2020).

[54] Stephen Ranshous, Steve Harenberg, Kshitij Sharma, and Nagiza F Samatova. 2016. A Scalable Approach for Outlier Detection in Edge Streams Using Sketch-based Approximations. In $S D M$.

[55] Markus Ring, Sarah Wunderlich, Deniz Scheuring, Dieter Landes, and Andreas Hotho. 2019. A survey of network-based intrusion detection data sets. Computers \& Security (2019).

[56] Peter J Rousseeuw and Katrien Van Driessen. 1999. A fast algorithm for the minimum covariance determinant estimator. Technometrics (1999).

[57] Saket Sathe and Charu C Aggarwal. 2016. Subspace Outlier Detection in Linear Time with Randomized Hashing. In ICDM.

[58] Neil Shah, Alex Beutel, Bryan Hooi, Leman Akoglu, Stephan Gunnemann, Disha Makhija, Mohit Kumar, and Christos Faloutsos. 2016. EdgeCentric: Anomaly Detection in Edge-Attributed Networks. In ICDMW.

[59] Iman Sharafaldin, Arash Habibi Lashkari, and Ali A Ghorbani. 2018. Toward Generating a New Intrusion Detection Dataset and Intrusion Traffic Characterization. In ICISSP.

[60] Lei Shi, Aryya Gangopadhyay, and Vandana P Janeja. 2015. STenSr: Spatiotemporal tensor streams for anomaly detection and pattern discovery. Knowledge and Information Systems (2015).

[61] Kijung Shin, Bryan Hooi, and Christos Faloutsos. 2016. M-zoom: Fast dense-block detection in tensors with quality guarantees. In ECMLPKDD.

[62] Kijung Shin, Bryan Hooi, Jisu Kim, and Christos Faloutsos. 2017. D-cube: Denseblock detection in terabyte-scale tensors. In WSDM. 
[63] Kijung Shin, Bryan Hooi, Jisu Kim, and Christos Faloutsos. 2017. DenseAlert: Incremental Dense-Subtensor Detection in Tensor Streams. KDD (2017).

[64] Hongyu Sun, Qiang He, Kewen Liao, Timos Sellis, Longkun Guo, Xuyun Zhang, Jun Shen, and Feifei Chen. 2019. Fast Anomaly Detection in Multiple MultiDimensional Data Streams. In BigData.

[65] Jimeng Sun, Dacheng Tao, and Christos Faloutsos. 2006. Beyond streams and graphs: dynamic tensor analysis. In $K D D$.

[66] Naftali Tishby, Fernando C Pereira, and William Bialek. 2000. The information bottleneck method. arXiv preprint physics/0004057 (2000). Duan, and Xing Li. 2005. Anomaly Internet Network Traffic Detection by Kernel Principle Component Classifier. In ISNN.

[68] Hanghang Tong and Ching-Yung Lin. 2011. Non-Negative Residual Matrix Factorization with Application to Graph Anomaly Detection. In SDM.

[69] Ravi Vinayakumar, Mamoun Alazab, KP Soman, Prabaharan Poornachandran, Ameer Al-Nemrat, and Sitalakshmi Venkatraman. 2019. Deep Learning Approach for Intelligent Intrusion Detection System. IEEE Access (2019).

[70] Wei Wang, Xiaohong Guan, Xiangliang Zhang, and Liwei Yang. 2006. Profiling program behavior for anomaly intrusion detection based on the transition and

[71] Wei Wang, Thomas Guyet, René Quiniou, Marie-Odile Cordier, Florent Masseglia, and Xiangliang Zhang. 2014. Autonomic intrusion detection: Adaptively detecting anomalies over unlabeled audit data streams in computer networks. KnowledgeBased Systems (2014).

[72] Yiwei Wang, Shenghua Liu, Minji Yoon, Hemank Lamba, Wei Wang, Christos Faloutsos, and Bryan Hooi. 2020. Provably Robust Node Classification via LowPass Message Passing. ICDM (2020).

[73] Audrey Wilmet, Tiphaine Viard, Matthieu Latapy, and Robin Lamarche-Perrin 2018. Degree-Based Outliers Detection Within IP Traffic Modelled as a Link Stream. 2018 Network Traffic Measurement and Analysis Conference (TMA) (2018).

[74] Audrey Wilmet, Tiphaine Viard, Matthieu Latapy, and Robin Lamarche-Perrin 2019. Outlier detection in IP traffic modelled as a link stream using the stability of degree distributions over time. Computer Networks (2019).

[75] Minji Yoon, Bryan Hooi, Kijung Shin, and Christos Faloutsos. 2019. Fast and Accurate Anomaly Detection in Dynamic Graphs with a Two-Pronged Approach. In $K D D$.

[76] Weiren Yu, Charu C Aggarwal, Shuai Ma, and Haixun Wang. 2013. On anomalous hotspot discovery in graph streams. In ICDM.

[77] Shuangfei Zhai, Yu Cheng, Weining Lu, and Zhongfei Zhang. 2016. Deep structured energy based models for anomaly detection. In ICML.

[78] Jiabao Zhang, Shenghua Liu, Wenjian Yu, Wenjie Feng, and Xueqi Cheng. 2019 EigenPulse: Detecting Surges in Large Streaming Graphs with Row Augmentation. In $P A K D D$.

[79] Shuo Zhou, Nguyen Xuan Vinh, James Bailey, Yunzhe Jia, and Ian Davidson. 2016. Accelerating online cp decompositions for higher order tensors. In KDD.

[80] Artur Ziviani, Antonio Tadeu A Gomes, Marcelo L Monsores, and Paulo SS Rodrigues. 2007. Network anomaly detection using nonextensive entropy. IEEE Communications Letters (2007).

[81] Bo Zong, Qi Song, Martin Renqiang Min, Wei Cheng, Cristian Lumezanu, Daeki Cho, and Haifeng Chen. 2018. Deep Autoencoding Gaussian Mixture Model for Unsupervised Anomaly Detection. In ICLR.
[67] Hanghang Tong, Chongrong Li, Jingrui He, Jiajian Chen, Quang-Anh Tran, Haixin frequency property of computer audit data. Computers \& Security (2006).

Table 5: Influence of temporal decay factor $\alpha$ on the ROCAUC in MSTREAM on CICIDS-DoS dataset.

\begin{tabular}{cc}
\hline$\alpha$ & ROC-AUC \\
\hline 0.1 & $0.9129 \pm 0.0004$ \\
0.2 & $0.9142 \pm 0.0009$ \\
0.3 & $0.9156 \pm 0.0006$ \\
0.4 & $0.9164 \pm 0.0014$ \\
0.5 & $0.9163 \pm 0.0005$ \\
0.6 & $0.917 \pm 0.0005$ \\
0.7 & $0.9196 \pm 0.0015$ \\
0.8 & $0.9235 \pm 0.0003$ \\
0.9 & $0.929 \pm 0.0003$ \\
0.95 & $0.9326 \pm 0.0006$ \\
\hline
\end{tabular}

Table 6: Influence of Output Dimensions on the ROC-AUC of MSTREAM-PCA, MSTREAM-IB, and MSTREAM-AE On KDDCUP99 dataset.

\begin{tabular}{rccc}
\hline Dimensions & MSTream-PCA & MSTream-IB & MSTream-AE \\
\hline 4 & 0.93 & 0.95 & 0.95 \\
8 & 0.94 & 0.95 & 0.93 \\
12 & 0.92 & 0.96 & 0.96 \\
16 & 0.87 & 0.96 & 0.96 \\
\hline
\end{tabular}

\section{DIMENSIONALITY REDUCTION}

For MSTREAM-IB, we used an online implementation, https://github. com/burklight/nonlinear-IB-PyTorch for the underlying Information Bottleneck algorithm with $\beta=0.5$ and the variance parameter set to a constant value of 1 . The network was implemented as a 2 layer binary classifier. For MSTREAM-AE, the encoder and decoder were implemented as single layers with ReLU activation.

Table 7 shows the network architecture of the autoencoder. Here $n$ denotes the batch size, and $d$ denotes the input data dimensions. The input data dimensions for each dataset are described in Section 5.

\section{Table 7: Autoencoder Architecture}

\begin{tabular}{ccc}
\hline Index & Layer & Output Size \\
\hline 1 & Linear & $n \times 12$ \\
2 & ReLU & $n \times 12$ \\
3 & Linear & $n \times d$ \\
\hline
\end{tabular}

\section{B INFLUENCE OF DIMENSIONS}

Table 6 shows the influence of the output dimensions on the ROCAUC for MSTREAM-PCA, MSTREAM-IB, and MSTREAM-AE KDDCUP99 dataset. We see that all methods are robust to the variation in output dimensions.
Table 5 shows the influence of the temporal decay factor $\alpha$ on the ROC-AUC for MSTREAM on CICIDS-DoS dataset. We see that $\alpha=$ 0.95 gives the maximum ROC-AUC for MSTREAM $(0.9326 \pm 0.0006)$, as also shown in Table 3. 
Table 8: MSTREAM-IB parameters for different datasets.

\begin{tabular}{rcccc}
\hline & \multicolumn{2}{c}{ MStream-IB } & \multicolumn{2}{c}{ MSTREAM-AE } \\
Dataset & Learning Rate & Epochs & Learning Rate & Epochs \\
\hline KDD & $1 \mathrm{e}-2$ & 100 & $1 \mathrm{e}-2$ & 100 \\
DoS & $1 \mathrm{e}-5$ & 200 & $1 \mathrm{e}-2$ & 1000 \\
UNSW & $1 \mathrm{e}-2$ & 100 & $1 \mathrm{e}-2$ & 100 \\
DDoS & $1 \mathrm{e}-3$ & 200 & $1 \mathrm{e}-3$ & 100 \\
\hline
\end{tabular}

\section{EVALUATING ROC-AUC IN A STREAMING MANNER}

Table 9 shows the ROC-AUC for MSTREAM-AE on KDDCUP99 when evaluated over the stream. The evaluation is done on all records seen so far and is performed after every $100 \mathrm{~K}$ records. We see that as the stream length increases, ROC-AUC for MSTREAM-AE converges to 0.96 , as also shown in Table 3 .
Table 9: Evaluating ROC-AUC of MSTREAM-AE in a streaming manner on KDDCUP99 dataset.

\begin{tabular}{rc}
\hline Stream Size & ROC-AUC \\
\hline $100 K$ & 0.912488 \\
$200 K$ & 0.895391 \\
$300 K$ & 0.855598 \\
$400 K$ & 0.934532 \\
$500 K$ & 0.965250 \\
$600 K$ & 0.953906 \\
$700 K$ & 0.947531 \\
$800 K$ & 0.961340 \\
$900 K$ & 0.973217 \\
$1000 K$ & 0.970212 \\
$1100 K$ & 0.967215 \\
$1200 K$ & 0.959664 \\
\hline
\end{tabular}

